\title{
Improved Firefly Algorithm for Unconstrained Optimization Problems
}

\author{
Khalil AL-Wagih* \\ Faculty of Computer science \& Information System, \\ Thamar University, Thamar, Republic of Yemen \\ E-mail: khalilwagih@gmail.com
}

\begin{abstract}
IFCH) is presented for solving unconstrained optimization problems. Several numerical simulation results show that the algorithm offers an efficient way to solve unconstrained optimization problems, and has a high convergence rate, high accuracy and robustness.
\end{abstract}

Keywords: Firefly algorithm; metaheuristic; optimization; chaos; unconstrained optimization

\section{INTRODUCTION}

The problem of finding the global optimum of a function with large numbers of local minima arises in many scientific applications. In typical applications, the search space is large and multi-dimensional. Many of these problems cannot be solved analytically, and consequently, they have to be addressed by numerical algorithms. Moreover, in many cases, global optimization problems are non-differentiable. Hence, the gradient-based methods cannot be used for finding the global optimum of such problems. To overcome these problems, several modern heuristic algorithms have been developed for searching near-optimum solutions to the problems. These algorithms can be classified into different groups, depending on the criteria being considered, such as population-based, iterative based, stochastic, deterministic, etc. Depending on the nature of the phenomenon simulated by the algorithms, the population-based heuristic algorithms have two important groups: Evolutionary Algorithms (EA) and swarm intelligence based algorithms.

Some of the recognized evolutionary algorithms are: Genetic Algorithms (GA) [1], Differential Evolution (DE) [2] and [3], Evolution Strategy (ES) [4] and Artificial Immune Algorithm (AIA) [5] etc. Some of the well known swarm intelligence based algorithms are: Particle Swarm Optimization (PSO), Ant Colony Optimization (ACO), Shuffled Frog Leaping (SFL), and Artificial Bee Colony (ABC) algorithms, etc. Besides the evolutionary and swarm intelligence based algorithms, there are some other algorithms which work on the principles of different natural phenomena. Some of them are: the Harmony Search (HS) algorithm, the Gravitational Search Algorithm (GSA), Biogeography-Based Optimization (BBO), the Grenade Explosion Method (GEM), the league championship algorithm and the charged system search .

This paper is organized as follows: after introduction, the original firefly algorithm is briefly introduced in section 2 . In section 3, the proposed algorithm is described, while the results are discussed in section 4. Finally, conclusions are presented in section 5 .

\section{FIREFLY ALGORITHM}

The Firefly Algorithm [FA] is one of many new optimization techniques that have been proposed over the past years. It was proposed by Yang in 2009 [6] and it has since then been applied in several applications bbecause of its few parameters to adjust, easy to understand, realize, and compute, it was applied to various fields, such as codebook of vector quantization [7], in-line spring-mass systems [8]; mixed variable structural optimization [9]; nonlinear grayscale image enhancement [10], travelling salesman problems [11], continuously cast steel slabs [12], promoting products online [13], nonconvex economic dispatch problems [14], chiller loading for energy conservation [15], stock market price forecasting [16], and multiple objectives optimization [17]. Although the algorithm has many similarities with other swarm based algorithms such as Particle Swarm Optimization [18], Artificial Bee Colony Optimization [19] and Ant Colony Optimization [6], the FA has proved to be much simpler both in concept and implementation and has better performance compared to the other techniques.

\subsection{Flashing behaviour of Fireflies}

The FA was based on the flashing patterns and behaviour patters of the fireflies. The fireflies use the flashing patterns to communicate with each other. Yang did not mimic their behaviour in full detail, but created a simplified algorithm based on the following three rules:

i. All fireflies are unisexual, so that one firefly will be attracted to other fireflies regardless of their sex;

ii. Attractiveness is proportional to the firefly's brightness; for any couple of flashing fireflies, the less bright one will move towards the brighter one; attractiveness is proportional to the brightness which decreases with increasing distance between fireflies; if there are no 
brighter fireflies than a particular firefly, this individual will move randomly in the space;

iii. The brightness of a firefly is somehow related to the analytical form of a cost function; for a maximization problem, brightness can be proportional to the value of the cost function; other forms of brightness can be defined in a similar matter to the fitness function in genetic algorithms.

\subsection{Attractiveness and Light Intensity}

In the algorithm, two important factors are involved: the variation of light intensity and the formulation of the attractiveness. For example, suppose that the attractiveness of a firefly is determined by its brightness, which in turn is associated with the encoded objective function, then the higher of the brightness and, the better the location and the more fireflies will be attracted to the direction. However, if the brightness is equal, the fireflies will move randomly. As light intensity and thus attractiveness decreases as the distance from the source increases, the variations of light intensity and attractiveness should be monotonically decreasing functions. In order to implement FA, there are some definitions:

Definition 1: the variation of light intensity;

We know, the light intensity varies according to the inverse square law

$$
I(r)=I_{s}{ }_{r^{2}}
$$

Where $\mathrm{I}(\mathrm{r})$ is the light intensity at a distance $\mathrm{r}$ and $\mathrm{I}_{\mathrm{s}}$ is the intensity at the source.

When the medium is given, the light intensity can be determined as follows:

$$
I(r)=I_{0} e^{-F}
$$

To avoid the singularity at $r=0$ in (1), the equations can be approximated in the following Gaussian form:

$$
I(r)=I_{0} e^{-Y^{2}}
$$

Where $\gamma$ is light absorption coefficient.

Definition 2: formulation of the attractiveness

As firefly attractiveness is proportional to the light intensity seen by adjacent fireflies, we can now define the attractiveness $\beta$ of a firefly by

$\beta=\beta_{0} e^{-W^{2}}$

Where $\beta_{0}$ is the attractiveness at $\mathrm{r}=0$.

Definition 3: formulation of location moving

$x_{\mathrm{i}}(t+1)=x_{\mathrm{i}}(t)+\beta\left(x_{j}(t)-x_{\mathrm{i}}(t)\right)+\alpha \varepsilon_{\mathrm{i}}$

Where $x_{\mathrm{i}}(t+1)$ is the position of $x_{\mathrm{i}}$ after $t+1$ times movements; $\alpha$ is the step parameter which varies between $[0,1] ; \varepsilon_{\mathrm{i}}$ is a random factor conforming Gaussian distribution between[0,1].

The basic steps of the FA are summarized as the pseudo code shown in Fig. 1 which consists of the three rules discussed above.

\begin{tabular}{l}
\hline firefly algorithm \\
Begin \\
Objective function $f(x), x=\left(x_{1}, \ldots, x_{d}\right)^{T}$ \\
\hline
\end{tabular}

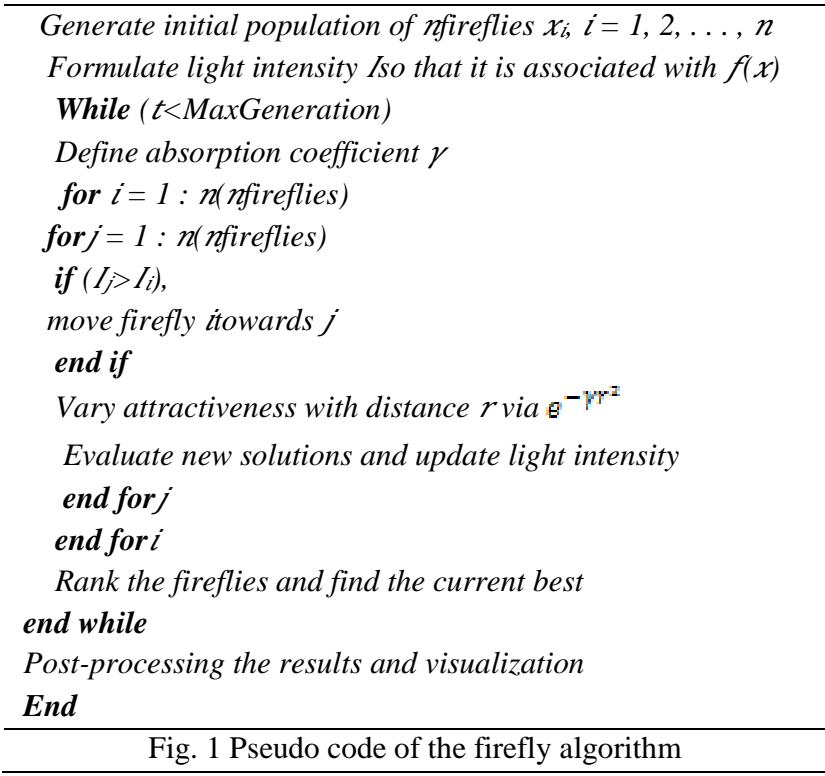

\section{THE PROPOSED ALGORITHM (IFCH) FOR UNCONSTRAINED OPTIMIZATION PROBLEMS}

Generating random sequences with a long period, and a good consistency is very important for easily simulating complex phenomena, sampling, numerical analysis, decision making and especially in heuristic optimization [20]. Its quality determines the reduction of storage and computation time to achieve the desired accuracy [21]. Chaos is a deterministic, random-like process found in nonlinear, dynamical system, which is non-period, non-converging and bounded. Moreover, it depends on its initial condition and parameters [22-24]. Applications of chaos in several disciplines including operations research, physics, engineering, economics, biology, philosophy and computer science[25-27].

Recently chaos is extended to various optimization areas because it can more easily escape from local minima and improve global convergence in comparison with other stochastic optimization algorithms [28-34]. Using chaotic sequences in FireflyAlgorithm can be helpfully improve the reliability of the global optimality, and also enhance the quality of the results.

In the proposed chaotic Firefly Algorithm, we used chaotic maps to tune the Firefly Algorithm parameters and improve the performance [20]. The steps of the proposed chaotic firefly algorithm for solving definite integral are as follows:

Step 1 Generate the initial population of fireflies,

$\left\{\mathrm{x}_{1}, \mathrm{x}_{2}, \mathrm{x}_{\mathrm{a}, \mathrm{x}, \mathrm{x}} \mathrm{x}_{\mathrm{n}}\right\}$

Step 2 Compute intensity for each firefly

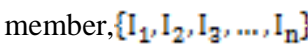

Step 3 Calculate the parameters $(\beta, 7)$ using the following Sinusoidal map[35]: 


$$
Y_{n+1}=\cos \left(k \cos ^{-1}\left(Y_{n}\right)\right) Y \in(-1,1)
$$

where $\mathrm{n}$ is the iteration number.

Step 4 Move each firefly $\mathrm{x}_{\mathrm{i}}$ towards other brighter fireflies. The position of each firefly is updated by

$$
x_{i}(t+1)=x_{i}(t)+\beta_{0} e^{-\gamma^{2}}\left(x_{j}(t)-x_{i}(t)\right)+\alpha \varepsilon_{i}
$$

Where $\alpha$ computed by the following randomness equation as shown below:

$$
\alpha^{i}=\alpha_{\max }-\left(\alpha_{\max }-\alpha_{\min }\right)\left(\frac{I_{\max }^{\mathrm{I}}-I_{\operatorname{man}}^{\mathrm{I}}}{I_{\max }^{\mathrm{i}}-I_{\min }^{\mathrm{i}}}\right)
$$

In this equation $\alpha^{\mathrm{I}}$ represents randomness parameters at cycle i. $\alpha_{\max }$ and $\alpha_{\min }$ represent maximum and minimum randomness parameters defined in the algorithm respectively. $I_{\max }^{\mathrm{I}}$ and $I_{\min }^{\mathrm{D}}$ represent maximum light intensity, minimum light intensity and mean value of light intensity of all fireflies at cycle i respectively.

Step 5 Update the solution set.

Step 6 Terminate if a termination criterion is fulfilled; otherwise go to Step 2.

\section{EXPERIMENTAL RESULTS}

Six well known test functions have been given to verify the weight of the proposed algorithm. The initial parameters are set at $n=40$; maximum iteration number $=100 ; \alpha_{\max }=0.8$; $\alpha_{\min }=0.1$. The results of IFCH algorithm are conducted from 50 independent runs for each problem. The selected chaotic map for all problems is the Sinusoidal map for $\beta_{8} 7$ values, and randomized for $\alpha$ values, whose equations is shown above.

All the experiments were performed on a Windows 7 Ultimate 64-bit operating system; processor Intel Core i7 760 running at $2.81 \mathrm{GHz} ; 8 \mathrm{~GB}$ of RAM and code was implemented in C\#.
Test problems are considered to extensively investigate the performance of the IFCH algorithm, and they are presented as follows:

The first is Sphere function, defined as

Min $\mathrm{f}_{1}=\sum_{i=1}^{N} x_{i}^{2}$

Where global optimum $\mathrm{x}^{*}=(0,0, \ldots, 0)$ and $\mathrm{f}\left(\mathrm{x}^{*}\right)=0$ for -100 $\leq \mathrm{x}_{\mathrm{i}} \leq 100$.

The second is Rosenbrock function, defined as

Min $\mathrm{f}_{2}=\sum_{i=1}^{N-1}\left(100\left(x_{i+1}-x_{i}^{i}\right)^{2}+\left(x_{i}-1\right)^{2}\right)$

Where global optimum $\mathrm{x}^{*}=(1,1, \ldots, 1)$ and $\mathrm{f}\left(\mathrm{x}^{*}\right)=0$ for -100 $\leq \mathrm{x}_{\mathrm{i}} \leq 100$.

The third is generalized Rastrigrin function, defined as

Min $\mathrm{f}_{3}=\sum_{i=1}^{N}\left(x_{i}^{2}-10 \cos \left(2 \pi x_{i}\right)+10\right)$

Where global optimum $\mathrm{x}^{*}=(0,0, \ldots, 0)$ and $\mathrm{f}\left(\mathrm{x}^{*}\right)=0$ for $-10 \leq$ $\mathrm{x}_{\mathrm{i}} \leq 10$.

The fourth function is as follows:

Min $\mathrm{f}_{4}=\sum_{i=1}^{N} z_{i}^{2}-450$

The fifth is generalized Griewank function, defined as

Min $\mathrm{f}_{5}=\frac{1}{4000} \sum_{i=1}^{N} x_{i}^{2}-\prod_{i=1}^{N} \cos \left(\frac{x_{i}}{\sqrt{i}}\right)+1$

Where global optimum $\mathrm{x}^{*}=(0,0, \ldots, 0)$ and $\mathrm{f}\left(\mathrm{x}^{*}\right)=0$ for -600 $\leq \mathrm{x}_{\mathrm{i}} \leq 600$.

The sixth is Schwefel's function, defined as

Min $\mathrm{f}_{6}=\sum_{i=1}^{N}\left|x_{i}\right|+\prod_{i=1}^{N}\left|x_{i}\right|$

Where global optimum $\mathrm{x}^{*}=(0,0, \ldots, 0)$ and $\mathrm{f}\left(\mathrm{x}^{*}\right)=0$ for -100 $\leq \mathrm{x}_{\mathrm{i}} \leq 100$.

Table 1 the solution of proposed algorithm and firefly algorithm

\begin{tabular}{|c|c|c|c|c|c|}
\hline Test Problem & Algorithm & Best & Worst & Mean & Standard Deviation \\
\hline \multirow{2}{*}{$f \mathbf{1}$} & FA & $6.0076 \mathrm{e}+001$ & $2.5205 \mathrm{e}+002$ & $1.7603 \mathrm{e}+002$ & $4.4359 \mathrm{e}+001$ \\
\cline { 2 - 7 } & IFCH & $2.2757 \mathrm{e}-010$ & $7.0950 \mathrm{e}-009$ & $1.8091 \mathrm{e}-009$ & $1.9488 \mathrm{e}-009$ \\
\hline \multirow{2}{*}{$\boldsymbol{f} \mathbf{}$} & FA & $9.6629 \mathrm{e}+004$ & $8.9231 \mathrm{e}+005$ & $3.3964 \mathrm{e}+005$ & $1.9909 \mathrm{e}+005$ \\
\cline { 2 - 7 } & IFCH & $2.4458 \mathrm{e}-001$ & $8.1098 \mathrm{e}+003$ & $5.3162 \mathrm{e}+002$ & $1.7208 \mathrm{e}+003$ \\
\hline \multirow{2}{*}{$\boldsymbol{f 3}$} & FA & $2.9511 \mathrm{e}+001$ & $5.2810 \mathrm{e}+001$ & $4.2476 \mathrm{e}+001$ & $5.7278 \mathrm{e}+000$ \\
\cline { 2 - 7 } & IFCH & $3.7993 \mathrm{e}-009$ & $9.9496 \mathrm{e}-001$ & $3.3166 \mathrm{e}-002$ & $1.8165 \mathrm{e}-001$ \\
\hline \multirow{2}{*}{$\boldsymbol{f 4}$} & FA & $-3.5043 \mathrm{e}+002$ & $-1.6889 \mathrm{e}+002$ & $-2.5642 \mathrm{e}+002$ & $4.4840 \mathrm{e}+001$ \\
\cline { 2 - 7 } & IFCH & $-4.5000 \mathrm{e}+002$ & $-4.5000 \mathrm{e}+002$ & $-4.5000 \mathrm{e}+002$ & $1.2822 \mathrm{e}-009$ \\
\hline \multirow{2}{*}{$\boldsymbol{f 5}$} & FA & $2.0566 \mathrm{e}+000$ & $3.2913 \mathrm{e}+000$ & $2.5946 \mathrm{e}+000$ & $3.2134 \mathrm{e}-001$ \\
\cline { 2 - 7 } & IFCH & $4.4744 \mathrm{e}-010$ & $1.3045 \mathrm{e}-001$ & $3.7959 \mathrm{e}-002$ & $3.8783 \mathrm{e}-002$ \\
\hline \multirow{2}{*}{$\boldsymbol{f 6}$} & FA & $3.1353 \mathrm{e}+001$ & $5.0629 \mathrm{e}+001$ & $4.2350 \mathrm{e}+001$ & $4.9070 \mathrm{e}+000$ \\
\cline { 2 - 7 } & IFCH & $3.6976 \mathrm{e}-005$ & $2.2687 \mathrm{e}-004$ & $8.1858 \mathrm{e}-005$ & $3.7660 \mathrm{e}-005$ \\
\hline
\end{tabular}




\section{CONCLUSIONS}

This paper introduced an improved Firefly Algorithm by blending with chaos for unconstrained optimization problems. The proposed algorithm employs a novel method for generating new solutions that enhances accuracy and convergence rate of FA. The proposed algorithm has been successfully applied to various benchmarking of unconstrained optimization problems. Case study results reveal that the proposed algorithm can find the global optimal solutions and is a powerful search algorithm for various unconstrained optimization problems.

\section{REFERENCES}

[1] H., Holland, Adaptation in Natural and Artificial Systems, University of Michigan Press,

Ann Arbor, USA (1975).

[2] R.Storn, and K.Price, "Differential evolution-a simple and efficient heuristic for global optimization over continuous spaces", J. Global Optim., 11, pp. 341-359 (1997).

[3] K.Price, , R. Storn, and A.Lampinen, , Differential Evolution-A Practical Approach to Global Optimization, Springer-Verlag, Berlin, Germany (2005).

[4] T.P.Runarsson, and X. Yao, "Stochastic ranking for constrained evolutionary optimization', IEEE Trans. Evol. Comput., 4(3), pp. 284 294(2000).

[5] L.J. Fogel, , A.J. Owens, and M.J.Walsh, , Artificial Intelligence Through Simulated Evolution, John Wiley, New York, USA (1966).

[6] X.-S. Yang, Nature-inspired metaheuristic algorithms: Luniver Press, 2010.

[7] M.-H. Horng and T.-W. Jiang, "The codebook design of image vector quantization based on the firefly algorithm," in Computational Collective Intelligence. Technologies and Applications, ed: Springer, 2010, pp. 438-447.

[8] R. Dutta, R. Ganguli, and V. Mani, "Exploring isospectral spring-mass systems with firefly algorithm," Proceedings of the Royal Society A: Mathematical, Physical and Engineering Science, vol. 467, pp. 3222-3240, 2011.

[9] A. H. Gandomi, X.-S. Yang, and A. H. Alavi, "Mixed variable structural optimization using firefly algorithm," Computers \& Structures, vol. 89, pp. 2325-2336, 2011.

[10] T. Hassanzadeh, H. Vojodi, and F. Mahmoudi, "Non-linear grayscale image enhancement based on firefly algorithm," in Swarm, Evolutionary, and Memetic Computing, ed: Springer, 2011, pp. 174181.

[11] G. K. Jati, "Evolutionary discrete firefly algorithm for travelling salesman problem," in Adaptive and Intelligent Systems, ed: Springer, 2011, pp. 393-403.

[12] O. K. K. L. JEKLENE, "Optimization of the Quality of Continuously Cast Steel Slabs Using the Firefly Algorithm," Materiali in tehnologije, vol. 45, pp. 347-350, 2011.

[13] H. Banati and M. Bajaj, "Promoting products online using firefly algorithm," in Intelligent Systems Design and Applications (ISDA), 2012 12th International Conference on, 2012, pp. 580-585.
X.-S. Yang, S. S. Sadat Hosseini, and A. H. Gandomi, "Firefly algorithm for solving non-convex economic dispatch problems with valve loading effect," Applied Soft Computing, vol. 12, pp. 11801186, 2012.

[15] L. d. S. Coelho and V. C. Mariani, "Improved firefly algorithm approach for optimal chiller loading for energy conservation," Energy and Buildings, 2012.

[16] A. Kazem, E. Sharifi, F. K. Hussain, M. Saberi, and O. K. Hussain, "Support vector regression with chaos-based firefly algorithm for stock market price forecasting," Applied Soft Computing, 2012.

[17] X.-S. Yang, "Multiobjective firefly algorithm for continuous optimization," Engineering with Computers, pp. 1-10, 2013.

[18] R. Poli, J. Kennedy, and T. Blackwell, "Particle swarm optimization," Swarm intelligence, vol. 1, pp. 33-57, 2007.

[19] P.-W. TSai, J.-S. Pan, B.-Y. Liao, and S.-C. Chu, "Enhanced artificial bee colony optimization," International Journal of Innovative Computing, Information and Control, vol. 5, pp. 5081-5092, 2009.

[20] B. Alatas, "Chaotic harmony search algorithms," Applied Mathematics and Computation, vol. 216, pp. 2687-2699, 2010

[21] W. Gong and S. Wang, "Chaos Ant Colony Optimization and Application," in Internet Computing for Science and Engineering (ICICSE), 2009 Fourth International Conference on, 2009, pp. 301-303.

[22] B. Alatas, "Chaotic bee colony algorithms for global numerical optimization," Expert Systems with Applications, vol. 37, pp. 5682-5687, 2010.

[23] A. Gandomi, X.-S. Yang, S. Talatahari, and A. Alavi, "Firefly algorithm with chaos," Communications in Nonlinear Science and Numerical Simulation, vol. 18, pp. 89-98, 2013.

[24] J. Mingjun and T. Huanwen, "Application of chaos in simulated annealing," Chaos, Solitons \& Fractals, vol. 21, pp. 933-941, 2004.

[25] L. d. S. Coelho and V. C. Mariani, "Use of chaotic sequences in a biologically inspired algorithm for engineering design optimization," Expert Systems with Applications, vol. 34, pp. 1905-1913, 2008.

[26] M. S. Tavazoei and M. Haeri, "Comparison of different one-dimensional maps as chaotic search pattern in chaos optimization algorithms," Applied Mathematics and Computation, vol. 187, pp. 10761085, 2007.

[27] R. Hilborn, Chaos and nonlinear dynamics: an introduction for scientists and engineers: oxford university press, 2000.

[28] D. He, C. He, L.-G. Jiang, H.-W. Zhu, and G.-R $\mathrm{Hu}$, "Chaotic characteristics of a one-dimensional iterative map with infinite collapses," Circuits and Systems I: Fundamental Theory and Applications, IEEE Transactions on, vol. 48, pp. 900-906, 2001.

[29] A. Erramilli, R. Singh, and P. Pruthi, Modeling packet traffic with chaotic maps: Citeseer, 1994.

[30] R. M. May, "Simple mathematical models with very complicated dynamics," in The Theory of Chaotic Attractors, ed: Springer, 2004, pp. 85-93.

[31] A. Wolf, "Quantifying chaos with Lyapunov exponents," Chaos, pp. 273-290, 1986. 
International Journal of Computer Applications Technology and Research

Volume 4- Issue 1, 77 - 81, 2015, ISSN:- 2319-8656

[32] R. L. Devaney, "An introduction to chaotic dynamical systems," 2003.

[33] C. Letellier, Chaos in nature vol. 81: World Scientific Publishing Company, 2013.

[34] R. Barton, "Chaos and fractals," The Mathematics Teacher, vol. 83, pp. 524-529, 1990.

[35] I. Fister, I. JrFister, X.-S. Yang, and J. Brest, "A comprehensive review of firefly algorithms," Swarm and Evolutionary Computation, 2013. 